\title{
Preferential association of prostate cancer cells expressing prostate specific membrane antigen to bone marrow matrix
}

\author{
SONALI P. BARWE ${ }^{*}$, RAYMOND S. MAUL ${ }^{1 *}$, JASON J. CHRISTIANSEN ${ }^{1}$, \\ GOPALAKRISHNAPILLAI ANILKUMAR ${ }^{1,4}$, CARLTON R. COOPER ${ }^{2}$, \\ DONALD B. KOHN ${ }^{3}$ and AYYAPPAN K. RAJASEKARAN ${ }^{1}$ \\ ${ }^{1}$ Department of Pathology and Laboratory Medicine, David Geffen School of Medicine, University of California, \\ Los Angeles, CA 90095; ${ }^{2}$ Department of Biological Sciences, University of Delaware, Newark, DE 19716; \\ ${ }^{3}$ Department of Molecular Microbiology and Immunology, Keck School of Medicine, \\ University of Southern California, Los Angeles, CA 90027, USA
}

Received September 5, 2006; Accepted October 25, 2006

\begin{abstract}
Prostate specific membrane antigen (PSMA) is a transmembrane glycoprotein expressed almost exclusively in prostatic epithelial cells. Expression of PSMA is elevated in prostate cancer, with levels closely correlated with disease grade. Although the highest levels of PSMA expression are associated with high-grade, hormone-refractory and metastatic prostate cancer, the significance of elevated PSMA expression in advanced prostate cancer has yet to be fully elucidated. We provide evidence that prostatic carcinoma cells expressing PSMA exhibit reduced motility and increased attachment when grown on a bone marrow matrix substrate. This phenomenon occurs via activation of focal adhesion kinase and provides the first evidence of a link between PSMA expression and prostate cancer metastasis to the bone.
\end{abstract}

\section{Introduction}

Prostate specific membrane antigen (PSMA) is a type II integral membrane glycoprotein with multiple putative functions within the cell (1). The large extracellular domain of PSMA shares a moderate degree of homology with the transferrin receptor and members of the M28 family of co-catalytic peptidases $(2,3)$. While the transferrin receptor has only a vestigial catalytic

Correspondence to: Dr Ayyappan K. Rajasekaran, Department of Pathology and Laboratory Medicine, Room 13-344 CHS, University of California, Los Angeles, CA 90095, USA

E-mail: arajasekaran@mednet.ucla.edu

${ }^{*}$ Contributed equally

Present address: ${ }^{4}$ Centre for Biotechnology, Amrita Vishwa Vidyapeetham, Amritapuri, Kollam, Kerala, India

Key words: prostate specific membrane antigen, bone marrow matrix, prostate cancer, motility, bone metastasis site, PSMA is known to possess two related enzymatic activities, referred to as folate hydrolase and $N$-acetylated $\alpha$-linked acidic dipeptidase (NAALADase) (4-6). In contrast to the large extracellular domain, the cytoplasmic tail of PSMA consists of just 19 amino acids (2). The short intracellular domain interacts with the actin cross-linking protein, filamin A (FLNa) and is involved in mediating endocytosis $(7,8)$.

As the name would suggest, expression of PSMA is predominantly restricted to cells of the prostatic epithelium $(9,10)$. PSMA is upregulated in practically all cases of prostate cancer, with expression levels directly proportional to disease grade $(11,12)$. The highest levels of PSMA expression are correlated with hormone-refractory and metastatic prostate cancer $(13,14)$. These advanced conditions represent a most insidious threat to the patient and are ultimately responsible for the lethality associated with prostate cancer. While localized disease is easily curable by surgery, viable treatment options are severely limited once tumor cells have colonized distant sites.

In order to metastasize, primary tumor cells must invade surrounding tissue, traverse extracellular matrix material and cross microvascular walls to enter the circulation (15). These cells then travel to distant sites where they must be able to colonize and clonally expand (15). Primary tumors of different origins demonstrate a tendency to metastasize to specific sites, where they possess a selective advantage. This is largely determined by intrinsic properties specific to the tumor cells and their interactions with particular organ microenvironments. Prostate carcinoma cells have an especially high propensity to metastasize to bone, with the vast majority of patients suffering from advanced disease presenting evidence of skeletal metastases $(15,16)$. Bone metastases are practically incurable and present profound morbidity to the patient, including bone fragility, increased susceptibility to fracture, and severe pain (17).

Antibodies against the extracellular domain of PSMA are currently being exploited as a means to detect and treat metastatic prostate cancer $(18,19)$. While such clinical strategies attempt to exploit the salient association between PSMA and advanced prostate cancer, the significance of elevated PSMA 
expression for tumor progression and metastasis is currently unknown. In this study, we provide evidence that PSMA expressing cells preferentially adhere to bone marrow matrix (BMM) through enhanced attachment involving activation of focal adhesion kinase (FAK). These observations could imply an etiological role for PSMA in promoting colonization and metastasis of prostate cancer cells to the bone.

\section{Materials and methods}

Cell culture and reagents. PC3 cells obtained from American Type Culture Collection (Manassas, VA) were cultured in RPMI (Life Technologies, Rockville, MD) supplemented with $10 \mathrm{mM}$ glutamine and $10 \%$ fetal bovine serum (FBS). Cells were maintained at $37^{\circ} \mathrm{C}$ with $5 \% \mathrm{CO}_{2}$ in a humidified atmosphere. The cDNA encoding full length PSMA was amplified by RT-PCR with total RNA isolated from LNCaP cells using TRIzol reagent (Invitrogen, Carlsbad, CA). The cDNA was cloned into a lentiviral vector system and used to generate PC3 cells homogenously expressing high levels of PSMA (PC3-PSMA). BMM was prepared as previously described (20). Suramin was purchased from Sigma-Aldrich (St. Louis, MO).

Immunofluorescence. Cells grown to $70 \%$ confluency on glass coverslips were fixed with either cold methanol or paraformaldehyde. Paraformaldehyde-fixed cells were permeablized with saponin and all specimens were subjected to immunofluorescence as described (21). After blocking with BSA, cells were incubated for $1 \mathrm{~h}$ with monoclonal antibody $(\mathrm{mAb})$ J591 against the extracellular domain of PSMA (22), washed, and counterstained with FITC-conjugated rabbit anti-mouse secondary antibody (Jackson ImmunoResearch Laboratories, West Grove, PA). Texas Red-phalloidin (Molecular Probes, Eugene, OR) was used to stain F-actin and visualize the actin cytoskeleton. To detect PSMA and FLNa colocalization, methanol-fixed cells were incubated with mAb E99 against PSMA (IgG3, kindly provided by Dr Neil Bander, Weill Medical College of Cornell University, New York) and mAb PM6/317 against FLNa (IgG1, Chemicon Internatinal Inc., Temecula, CA), washed, and counter-stained with Texas red conjugated IgG3-specific anti-mouse antibody (Jackson ImmunoResearch Laboratories) and FITC-conjugated rabbit anti-mouse secondary antibody (IgG1-specific).

Wound-healing migration assay. PC3 and PC3-PSMA cells were cultured to $\sim 80 \%$ confluency. Cell monolayers were washed in PBS and wounded by scoring the bottom of the culture plate with a sterile pipette tip. The wounded cells were gently washed twice in PBS and returned to RPMI supplemented with $10 \%$ FBS. Four defined areas on each dish were recorded at time zero by phase-contrast microscopy and monitored at 3- and 6-h intervals. The distance migrated for each defined area was measured and the mean distance calculated for the four separate measurements, and each of the four defined areas was averaged for a final migration value.

Quantification of cell-ECM attachment. PC3 and PC3-PSMA cells $\left(5.5 \times 10^{5}\right)$ were cultured for $24 \mathrm{~h}$ in $35-\mathrm{mm}$ diameter
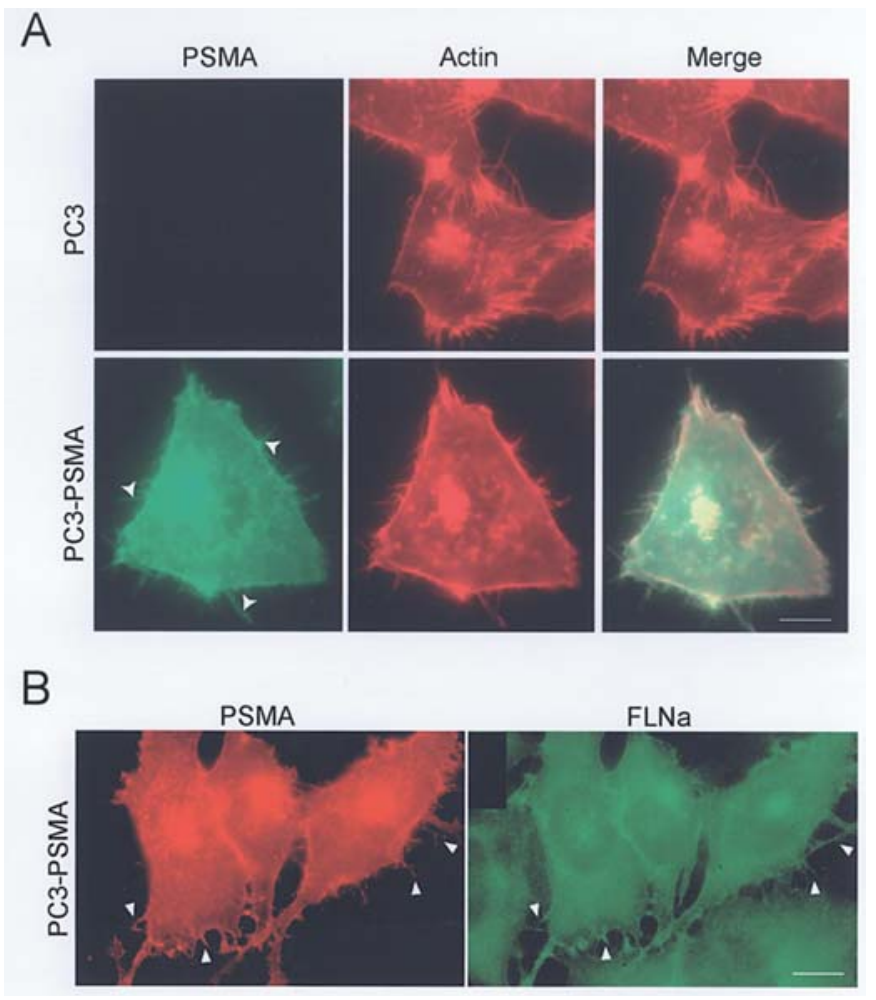

Figure 1. Localization of PSMA and FLNa to actin-rich filopodia in PC3PSMA cells. (A) PC3 or PC3-PSMA cells were incubated with mAb J591 and stained with FITC-conjugated anti-mouse antibody to visualize PSMA. F-actin was stained with Texas Red-conjugated phalloidin. The merged images are shown in the right column. Arrowheads indicate PSMA staining on the filopodia. (B) PC3-PSMA cells were methanol-fixed, and incubated with mAb E99 (IgG3) and anti-FLNa (IgG1) antibodies, and stained with Texas Red-conjugated anti-mouse IgG3 and FITC-conjugated anti-mouse IgG1 antibodies. Note that PSMA and FLNa localize to the filopodia (arrowheads). The images were overexposed to show the thin filopodia. Bar, $20 \mu \mathrm{m}$.

dishes on the appropriate matrix-coated plates. Dishes were coated with the indicated substrates; fibronectin, collagen, or soluble bone marrow matrix (BMM). Fibronectin $(4 \mu \mathrm{g} / \mathrm{ml})$ or collagen type $\mathrm{I}(10 \mu \mathrm{g} / \mathrm{ml})$ was incubated overnight at $4^{\circ} \mathrm{C}$ in PBS or $50 \mathrm{mM}$ acetic acid, respectively. For BMM, $0.1 \mathrm{ml}$ of BMM $(100 \mu \mathrm{g} / \mathrm{ml})$ in aqueous solution was spread onto culture dishes and allowed to dry under sterile conditions immediately before use. Cells were washed with serum-free RPMI and incubated with $0.01 \%$ trypsin, $10.6 \mathrm{mM}$ EDTA in PBS until cells started to detach. This variable time was determined for each substrate. Cells were subsequently washed with serum-free RPMI and fixed in $20 \%$ methanol containing $0.5 \%$ crystal violet for $20 \mathrm{~min}$. After washing multiple times with distilled water, the stain was extracted with $1 \mathrm{ml}$ of $10 \%$ acetic acid, and quantified at $600 \mathrm{~nm}$ on a spectrophotometer to produce a value representing the number of cells that remained attached after trypsin treatment.

Immunoprecipitation and Western blotting. PC3 and PC3PSMA cells were trypsinized and plated on either uncoated or BMM-coated tissue culture dishes for $5 \mathrm{~h}$. The cells were washed once with ice-cold PBS and lysed in $0.75 \mathrm{ml}$ of ice-cold RIPA buffer. Lysates were scraped, sonicated, and subjected to centrifugation at 13,200 rpm for $10 \mathrm{~min}$. Supernatants were 
A

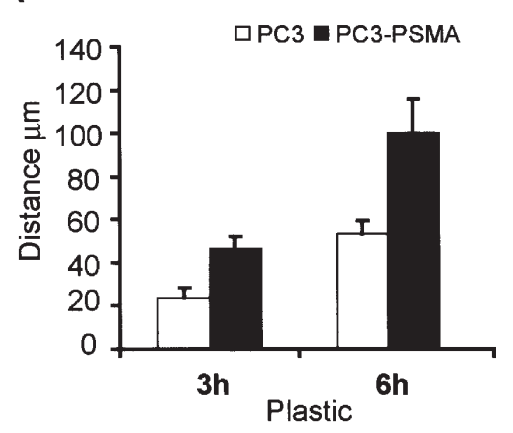

C

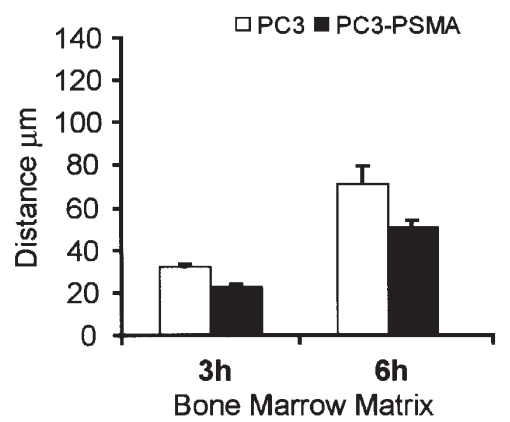

B

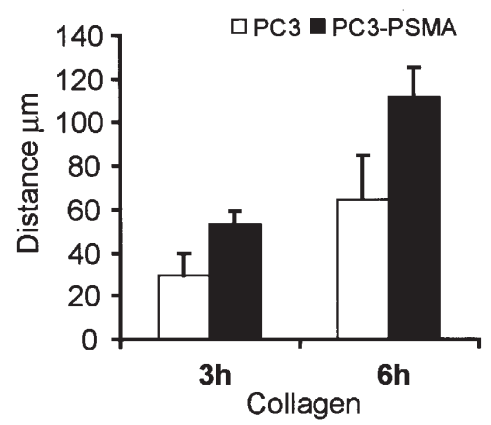

D

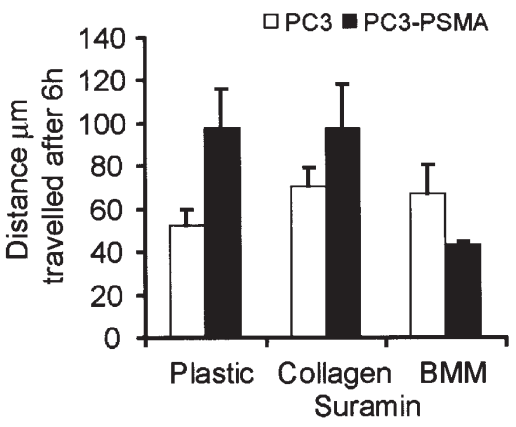

Figure 2. The effect of PSMA expression on the migration of PC3 cells on various substrates. A subconfluent monolayer of either PC3 or PC3-PSMA cells was wounded and the wound size was recorded at time zero, and after 3 and $6 \mathrm{~h}$ of incubation at $37^{\circ} \mathrm{C}$. The total distance migrated in microns was calculated for 3 and $6 \mathrm{~h}$ on tissue culture plastic (A), collagen-coated dishes (B), and BMM-coated dishes (C). (D) The cells were incubated with 100 nM suramin after wounding in order to inhibit the NAALADase activity of PSMA. The distance migrated $6 \mathrm{~h}$ later on tissue culture plastic, collagen, and BMM-coated dishes is calculated. Data represent mean values plus standard errors from three (A-C) or two (D) independent experiments.

transferred to protein A-agarose beads pre-coupled with $1 \mu \mathrm{g} / \mathrm{ml}$ of polyclonal anti-FAK (C20, Santa Cruz Biotechnology Inc., Santa Cruz, CA) antibody. Proteins were immunoprecipitated at $4^{\circ} \mathrm{C}$ for $16 \mathrm{~h}$. Immunoprecipitates were washed three times with RIPA buffer and extracted with $2 X$ SDS-PAGE sample buffer by boiling for $5 \mathrm{~min}$. Samples were separated by SDSPAGE and subjected to immunoblot analysis. Phosphorylated FAK protein was detected by incubation with anti-phospho tyrosine PY20 mouse monoclonal antibody (BD Transduction Laboratories, Lexington, KY) in 5\% BSA in Tris-buffered saline containing $0.1 \%$ Tween-20. Bands were quantified by densitometry using Bio-Rad (Hercules, CA) image software Quantity One.

\section{Results}

PSMA localizes to actin-rich filopodia. Previous studies from our laboratory showed an association of the cytoplasmic tail of PSMA with the actin cross-linking protein FLNa (7). FLNa can regulate cell motility via the actin cytoskeleton and associated signal transduction pathways (23). FLNa is also known to localize to the filopodia (24), which are actinrich structures implicated in the control of cell motility (25). Therefore, we hypothesized that PSMA might have a role in the motility of prostate cancer cells. Immunofluorescence analysis revealed that PSMA distinctly co-localized with actin at the filopodia in PC3-PSMA cells (Fig. 1A, arrowheads). Control PC3 cells also displayed filopodia indicating that PSMA expression is not necessary to induce filopodia in PC3 cells. Further, PSMA co-localized with FLNa at the filopodia in PC3-PSMA cells (Fig. 1B, arrowheads). These results indicated that PSMA could be localized to sites of actin polymerization associated with cell motility.

PSMA expression affects the motility of prostate cancer cells. To obtain insights into whether PSMA expression is associated with cell motility, we tested the motility of PC3-PSMA cells using a wound-healing migration assay, which is commonly used to monitor motility in carcinoma cells. Since prostate cancer cells are known to metastasize to the bone, and PSMA expression increases in advanced and metastatic prostate cancer, we sought to test the motility of control PC3 and PC3-PSMA cells grown on human BMM, as well as on collagen coated and uncoated tissue culture plastic dishes. The motility of PC3-PSMA cells on plastic and collagen coated dishes was increased 1.9- and 1.7-fold respectively at both the 3- and 6-h time-points, when compared to control PC3 cells (Fig. 2A and B). In contrast, the motility of PSMAexpressing cells was reduced by $30 \%$ on BMM coated dishes (Fig. 2C). These results indicated that expression of PSMA could affect the motility of cancer cells, and that this effect was substrate-dependent.

Next we tested whether the enzymatic activity of PSMA is necessary to control cell motility. Pharmacological inhibition of PSMA enzymatic activity should decrease motility on plastic and collagen, and increase motility on BMM, if the regulation of cell motility by PSMA was dependent on its enzymatic activity. After wounding, cells were treated with 
A

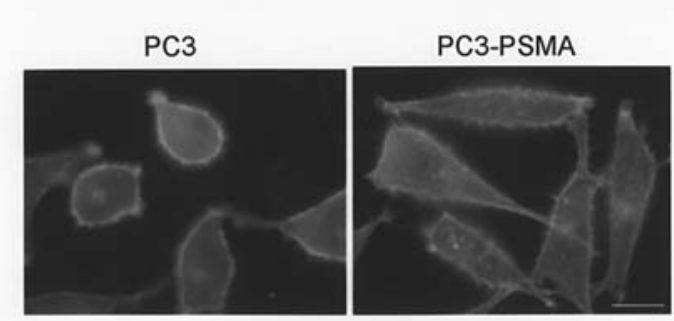

B
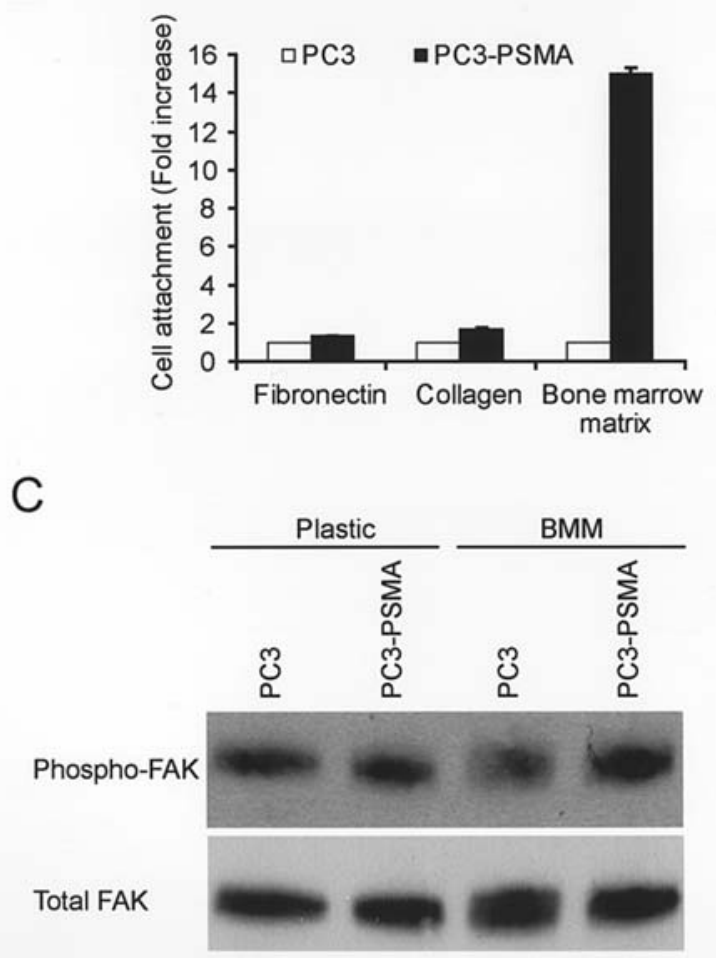

Figure 3. PC3-PSMA cells grown on BMM spread faster, exhibit greater attachment, and have increased FAK activation when compared to PC3 cells. (A) Cell morphology and actin cytoskeleton for PC3 and PC3-PSMA cells was evaluated $4 \mathrm{~h}$ following adhesion to BMM-coated coverslips. Cells were stained using Texas Red-conjugated phalloidin. (B) The adhesive properties of PC3 and PC3-PSMA cells were compared by monitoring trypsin-induced cell detachment from fibronectin, collagen, and BMMcoated dishes. The absorbance corresponding to the number of PC3 cells remaining attached after a fixed period of time was set equal to one for each substrate. Data represent mean values plus standard error from three independent experiments. (C) The tyrosine phosphorylation status of FAK was monitored $5 \mathrm{~h}$ after adhesion to uncoated tissue culture plastic dishes or to BMM-coated dishes. FAK was immunoprecipitated with a specific polyclonal antibody and immunoblotted with anti-phosphotyrosine antibody. The same blot was stripped and reprobed with anti-FAK antibody to determine the levels of total FAK protein.

$100 \mathrm{nM}$ suramin, a specific inhibitor of NAALADase activity (26). No change in the motility of the cells on plastic, collagen, or BMM was observed with suramin treatment (Fig. 2D). This result indicated that the enzymatic activity of PSMA might not be essential for the motility function of PSMA.

PC3-PSMA cells show spreading and preferential attachment on BMM when compared with PC3 cells. We next investigated the morphology of the control PC3 and PC3-PSMA cells grown on BMM. For this purpose, cells were plated for $4 \mathrm{~h}$ on BMM-coated coverslips, fixed, and stained with fluorescent phalloidin to visualize the actin cytoskeleton. Strikingly, the PC3-PSMA cells showed increased cell spreading with more elongated morphology compared to PC3 cells (Fig. 3A), which appeared more or less round. This morphological difference strongly indicated that PC3-PSMA cells are able to spread and adhere faster on BMM.

Cell motility involves several spatially and temporally coordinated processes that include disruption of focal adhesions, disassembly of cell substratum adhesion, formation of new focal contacts, and actin polymerization to drive membrane protrusion (27). Thus, it appears that increased motility depends on weak adhesion of cells to the substratum, whereas decreased motility would result from stronger adhesion. Given the different effects that PSMA expression and various ECMs had on cell motility, we next investigated the attachment properties of PC3 and PC3-PSMA cells by testing the resistance of cells to trypsin-induced detachment. Near confluent PC3 and PC3-PSMA cells were grown on fibronectin, collagen, or BMM for one day before being incubated with a low dose of trypsin solution. PC3-PSMA cells grown on fibronectin or collagen showed slightly elevated attachment compared to control PC3 cells when challenged with trypsin. However, there was a 15-fold increase in the adhesion of PC3-PSMA cells to BMM when compared to PC3 cells (Fig. 3B). This striking result indicated that PC3 cells expressing PSMA attached preferentially to the BMM.

Increased tyrosine phosphorylation status of FAK in PC3PSMA cells on BMM. Attachment of cells to the substratum is regulated in part by FAK, which is phosphorylated in response to integrin engagement. The activation of FAK-associated signaling complexes can promote the formation or turnover of focal contacts (28). Therefore, we compared the levels of tyrosine-phosphorylated FAK in PC3 and PC3-PSMA cells grown on plastic or BMM for $5 \mathrm{~h}$. We consistently observed a 1.6-fold increase in tyrosine-phosphorylated FAK in PC3-PSMA cells grown on BMM, while there was no change in phosphorylated FAK in cells grown on tissue culture plastic. This observation of increased FAK activity in PC3-PSMA cells plated on BMM is consistent with PSMA-expressing cells preferentially associating with, and being stimulated by the BMM.

\section{Discussion}

In the current study, we demonstrated that ectopic expression of PSMA in a prostate cancer cell line resulted in altered cell motility and adherence. This phenomenon was highly dependent upon the substratum onto which the cells were plated. PSMA expressing cells exhibited increased attachment relative to control cells when grown on BMM as against fibronectin or collagen substrates. Furthermore, PSMA expressing cells showed decreased cell motility, and an increased propensity for cell spreading and attachment on BMM that correlated with elevated tyrosine phosphorylation of FAK. These results suggest a potential role for PSMA in skeletal metastasis of prostate cancer cells. To our knowledge, this study is the first to implicate a role for PSMA in promoting metastatic disease. 
Our data suggest that expression of PSMA can induce either an increase or a decrease in cell motility, depending upon the substratum to which the cells are exposed. This seemingly paradoxical situation is not without precedent. For example, ECM-dependent regulation of cell migration has been demonstrated in case of Tiam1/Rac signaling. Although Tiam1/Rac signaling enhances migration of cells grown on collagen, the same signaling pathway effectively inhibits cell migration on laminin or fibronectin substrates (29). These patterns of matrix-dependent regulation of cell motility can be explained on the basis of expression or activation of substrate-specific signaling molecules. Therefore, depending on the signals derived from the ECM, it is possible that PSMA expression might activate signaling pathways that either stimulate or inhibit cell migration.

Since PSMA possesses peptidase activity, it was interesting to determine if the enzymatic activity of PSMA was involved in the motility regulation. When the enzymatic activity of PSMA was pharmacologically inhibited with suramin, there was no change in cell motility on all substrates tested. Moreover, it has been shown that the concentration of phosphate in the culture medium used is inhibitory to PSMA enzymatic activity (30). Thus, the enzymatic activity of PSMA is not likely to be involved in its motility function. Interestingly, a recent study indicated that inhibition of PSMA enzymatic activity increased the invasive potential of prostate cancer cells into Matrigel (31). However, evidence suggests that cell motility and invasion are regulated by different mechanisms. For example, it has been shown that $\alpha$-catenin is required for IGF-I-induced cellular migration but not for invasion (32). Taken together, these results (31) suggest that PSMA might modulate invasion by its enzymatic activity, and motility via protein-protein interactions. Future experiments are needed to further confirm these intriguing observations.

Alternatively, the mechanism responsible for decreased motility and increased attachment of cells on BMM could involve alterations in integrin signaling. Integrin-mediated signaling is proposed to play critical roles in cell growth and migratory processes by establishing and disrupting adhesive interactions between cancer cells and components of the ECM $(33,34)$. BMM represents a complex substratum that is primarily composed of soluble matrix proteins, including fibronectin, laminin, and proteoglycan (35). It is possible that the extracellular domain of PSMA might associate with components of this complex substratum leading to activation of integrin-mediated signaling. This signaling can cause activation of FAK resulting in reduced motility on BMM. The association of PSMA with FLNa might be involved in this process (8). Several studies have demonstrated that FLNa plays a pivotal role in cell migration (36). FLNa binding to $\beta$-integrin restricts integrin-dependent cell migration by inhibiting transient membrane protrusion and cell polarization (36). Thus, PSMA interacting with FLNa on the cytoplasmic face, and binding to a specific component of BMM via its extracellular domain might activate integrin signaling leading to increased FAK phosphorylation, and preferential association of PSMA expressing cells on BMM.

The effect of PSMA on cell motility and attachment could have important implications related to prostate cancer metastasis to bone. The formation of such metastatic lesions is a multi-step process that requires primary tumors to invade their surrounding benign tissues and escape from the prostate gland. These cells must then intravasate through micorvascular walls, enter into the circulation, and subsequently extravasate and colonize the bone (15). Once the prostate cancer cells reach the bone, factors in the bone microenvironment promote cell growth and bone remodeling. Integrins are proposed to play critical roles in these growth and migratory processes $(33,34)$. In particular, metastasis relies upon the establishment and disruption of adhesive interactions between cancer cells and the ECM mediated by integrins. ECM-dependent differential regulation of motility by PSMA might be advantageous for prostate cancer metastasis to the bone. The increased motility associated with PSMA expression might initially promote migration of prostate cancer cells through ECM at the site of the primary tumor. These cells would be accessible to vasculature and transit to distal sites, including the bones. Once there, the increased activation of FAK in response to contact with BMM would promote attachment of PSMA expressing cells and presumably facilitate colonization of the bone. Thus, our results propose a novel role for PSMA in the regulation of cell motility leading to the metastasis of prostate cancer cells to the bone. Experiments are in progress in our laboratory to elucidate the mechanism responsible for the increased cell adhesion and to identify components of BMM that are responsible for this phenomenon.

\section{Acknowledgements}

This work was supported by the Department of Defense W81XWH-04-1-0113 (AKR) and the University of Michigan Prostate Spore Grant CA69568. We thank Drs Neil Bander (Weill Medical College, Cornell University, NY) for providing mAbs J591, E99 and Kenneth Pienta (University of Michigan, Ann Arbor, MI) for the bone marrow matrix.

\section{References}

1. Rajasekaran AK, Anilkumar G and Christiansen JJ: Is prostatespecific membrane antigen a multifunctional protein? Am J Physiol Cell Physiol 288: C975-C981, 2005.

2. Israeli RS, Powell CT, Fair WR and Heston WD: Molecular cloning of a complementary DNA encoding a prostate-specific membrane antigen. Cancer Res 53: 227-230, 1993.

3. Rawlings ND and Barrett AJ: Structure of membrane glutamate carboxypeptidase. Biochim Biophys Acta 1339: 247-252, 1997.

4. Mahadevan D and Saldanha JW: The extracellular regions of PSMA and the transferrin receptor contain an aminopeptidase domain: implications for drug design. Protein Sci 8: 2546-2549, 1999

5. Pinto JT, Suffoletto BP, Berzin TM, et al: Prostate-specific membrane antigen: a novel folate hydrolase in human prostatic carcinoma cells. Clin Cancer Res 2: 1445-1451, 1996.

6. Carter RE, Feldman AR and Coyle JT: Prostate-specific membrane antigen is a hydrolase with substrate and pharmacologic characteristics of a neuropeptidase. Proc Natl Acad Sci USA 93: 749-753, 1996.

7. Anilkumar G, Rajasekaran SA, Wang S, Hankinson O, Bander NH and Rajasekaran AK: Prostate-specific membrane antigen association with filamin A modulates its internalization and NAALADase activity. Cancer Res 63: 2645-2648, 2003.

8. Rajasekaran SA, Anilkumar G, Oshima E, et al: A novel cytoplasmic tail MXXXL motif mediates the internalization of prostate-specific membrane antigen. Mol Biol Cell 14: 4835-4845, 2003. 
9. Horoszewicz JS, Kawinski E and Murphy GP: Monoclonal antibodies to a new antigenic marker in epithelial prostatic cells and serum of prostatic cancer patients. Anticancer Res 7: 927-935, 1987.

10. Israeli RS, Powell CT, Corr JG, Fair WR and Heston WD: Expression of the prostate-specific membrane antigen. Cancer Res 54: 1807-1811, 1994.

11. Silver DA, Pellicer I, Fair WR, Heston WD and Cordon-Cardo C: Prostate-specific membrane antigen expression in normal and malignant human tissues. Clin Cancer Res 3: 81-85, 1997.

12. Ross JS, Sheehan CE, Fisher HA, et al: Correlation of primary tumor prostate-specific membrane antigen expression with disease recurrence in prostate cancer. Clin Cancer Res 9: 6357-6362, 2003.

13. Wright GL Jr, Grob BM, Haley C, et al: Upregulation of prostatespecific membrane antigen after androgen-deprivation therapy Urology 48: 326-334, 1996.

14. Sweat SD, Pacelli A, Murphy GP and Bostwick DG: Prostatespecific membrane antigen expression is greatest in prostate adenocarcinoma and lymph node metastases. Urology 52: 637-640, 1998.

15. Yin JJ, Pollock CB and Kelly K: Mechanisms of cancer metastasis to the bone. Cell Res 15: 57-62, 2005.

16. Rubin MA, Putzi M, Mucci N, Smith DC, Wojno K, Korenchuk S and Pienta KJ: Rapid ('warm') autopsy study for procurement of metastatic prostate cancer. Clin Cancer Res 6: 1038-1045, 2000.

17. Mundy GR: Metastasis to bone: causes, consequences and therapeutic opportunities. Nat Rev Cancer 2: 584-593, 2002.

18. Nanus DM, Milowsky MI, Kostakoglu L, Smith-Jones PM, Vallabahajosula S, Goldsmith SJ and Bander NH: Clinical use of monoclonal antibody HuJ591 therapy: targeting prostate specific membrane antigen. J Urol 170: S84-S89, 2003.

19. Bander NH, Trabulsi EJ, Kostakoglu L, et al: Targeting metastatic prostate cancer with radiolabeled monoclonal antibody J591 to the extracellular domain of prostate specific membrane antigen. J Urol 170: 1717-1721, 2003.

20. Cooper CR, McLean L, Walsh M, Taylor J, Hayasaka S, Bhatia J and Pienta KJ: Preferential adhesion of prostate cancer cells to bone is mediated by binding to bone marrow endothelial cells as compared to extracellular matrix components in vitro. Clin Cancer Res 6: 4839-4847, 2000.

21. Barwe SP, Anilkumar G, Moon SY, Zheng Y, Whitelegge JP, Rajasekaran SA and Rajasekaran AK: Novel role for $\mathrm{Na}, \mathrm{K}$ ATPase in phosphatidylinositol 3-kinase signaling and suppression of cell motility. Mol Biol Cell 16: 1082-1094, 2005.

22. Liu H, Moy P, Kim S, et al: Monoclonal antibodies to the extracellular domain of prostate-specific membrane antigen also react with tumor vascular endothelium. Cancer Res 57: 3629-3634, 1997.
23. Stossel TP, Condeelis J, Cooley L, Hartwig JH, Noegel A, Schleicher M and Shapiro SS: Filamins as integrators of cell mechanics and signalling. Nat Rev Mol Cell Biol 2: 138-145, 2001

24. Ohta Y, Suzuki N, Nakamura S, Hartwig JH and Stossel TP: The small GTPase RalA targets filamin to induce filopodia. Proc Natl Acad Sci USA 96: 2122-2128, 1999.

25. Chodniewicz D and Klemke RL: Guiding cell migration through directed extension and stabilization of pseudopodia. Exp Cell Res 301: 31-37, 2004.

26. Slusher BS, Tiffany CW, Merion A, Lapidus RG and Jackson PF: Suramin potently inhibits the enzymatic activity of PSM. Prostate 44: 55-60, 2000 .

27. Ridley AJ, Schwartz MA, Burridge K, et al: Cell migration: integrating signals from front to back. Science 302: 1704-1709, 2003.

28. Parsons JT: Focal adhesion kinase: the first ten years. J Cell Sci 116: 1409-1416, 2003.

29. Sander EE, van Delft S, ten Klooster JP, Reid T, van der Kammen RA, Michiels F and Collard JG: Matrix-dependent Tiam1/Rac signaling in epithelial cells promotes either cell-cell adhesion or cell migration and is regulated by phosphatidylinositol 3-kinase. J Cell Biol 143: 1385-1398, 1998.

30. Slusher BS, Vornov JJ, Thomas AG, et al: Selective inhibition of NAALADase, which converts NAAG to glutamate, reduces ischemic brain injury. Nat Med 5: 1396-1402, 1999.

31. Ghosh A, Wang X, Klein E and Heston WD: Novel role of prostate-specific membrane antigen in suppressing prostate cancer invasiveness. Cancer Res 65: 727-731, 2005.

32. Andre F, Janssens B, Bruyneel E, van Roy F, Gespach C, Mareel $\mathrm{M}$ and Bracke $\mathrm{M}$ : Alpha-catenin is required for IGF-Iinduced cellular migration but not invasion in human colonic cancer cells. Oncogene 23: 1177-1186, 2004.

33. Slack-Davis JK and Parsons JT: Emerging views of integrin signaling: implications for prostate cancer. J Cell Biochem 91: 41-46, 2004.

34. Edlund M, Sung SY and Chung LW: Modulation of prostate cancer growth in bone microenvironments. J Cell Biochem 91: 686-705, 2004.

35. Peters C, Budde CL, Breon TA, Kuper A and Kim J: Ovine bone marrow extracellular matrix and soluble protein extraction: fetuin amino terminus microheterogeneity. Am J Med Sci 309: 285-294, 1995.

36. Calderwood DA, Huttenlocher A, Kiosses WB, Rose DM, Woodside DG, Schwartz MA and Ginsberg MH: Increased filamin binding to beta-integrin cytoplasmic domains inhibits cell migration. Nat Cell Biol 3: 1060-1068, 2001. 